Iraqi Journal of Information and Communications Technology(IJICT)

Conference Series: The $1^{\text {st }}$ Conference of Applied Researches

in Information Engineering(ARIE2021), 2021

ISSN:2222-758X

e-ISSN:2789-7362

\title{
IMAGE RETRIEVAL BASED ON DISCRETE CURVELET TRANSFORM
}

\author{
Zahraa H. Al-Obaide ${ }^{1}$, Ayad A. Al-Ani ${ }^{2}$ \\ 1,2 College of Information Engineering, Al-Nahrain University, Baghdad, Iraq \\ Zahraaalobaide97@gmail.com ${ }^{1}$, ayad.abdulaziz@ coie-nahrain.edu.iq ${ }^{2}$ \\ Received:4/5/2021, Accepted:1/6/2021
}

\begin{abstract}
Content-Based Image Retrieval (CBIR) is a process for searching an image according to the content or feature within the image. Nowadays, most image retrieval applications have been developed to meet these needs, so this application will provide comfort in introducing and searching for an image. In this paper, we proposed an automatic image retrieval method that significantly impacts the images. Our proposed method outperforms current CBIR systems. The obtained results of our query image by using CBIR method have been compared with all the database images using Euclidean distance. The system was performed with ten different classes of 1000 images using a coral database. From our results, we find that the best results were obtained when we used higher decomposition levels with improving the performance of precision. Also, we conclude that the best results have been obtained when we used a median filter with accuracy reached $99 \%$.
\end{abstract}

keywords: Image retrieval, CBIR, Curvelet transform, Feature extraction.

\section{INTRODUCTION}

Images make websites more interesting, and humans can process and understand images faster than reading text. At present, the number of images uploaded to the internet has increased dramatically. Many of these image collections pose increasing technical challenges for computer systems to manage and search these image data effectively [1]. Therefore, seeking efficient image retrieval mechanisms has piqued a wide area of interest for researchers. Methods of image retrieval can be categorized into two approaches [2]. The first one is based on a search by metadata known as Text Base Image Retrieval (TBIR), and The second approach is based on Content information in the image, also known as Content-Based Image Retrieval (CBIR). In the TBIR method, users use a keyword or description of images as a query to retrieve images that are related to the keyword [3]. Three main disadvantages of the TBIR for a vast database. First, there is inconsistency in labelling by different annotators due to different understanding of image contents. Second, there is a high probability of error occurs during the image tagging process when the database is large [4]. Third, it takes a lot of time to annotate each image in an extensive database and makes the process subjective [5]. As a result, TBIR cannot achieve a high level of efficiency and effectiveness. CBIR is also known as Query By Image Content (QBIC) [4], presents a flexible way to index images automatically based on the visual content of images (i.e., color, texture, and shape). The term CBIR appeared in the early 1990s [6]. A typical block diagram for content-based image retrieval is shown in Fig. 1 [7] . The main idea of CBIR is to find the most similar images with the query image from a dataset using distance metrics [8]. The CBIR system gives the consumer greater flexibility by offering an interactive environment. Users of the CBIR system are not constrained by any particular language. The system's language independence enables it to serve any user [4]. The CBIR technology has been used in many applications such as fingerprint identification, biodiversity information systems, digital libraries, crime prevention, and medicine. Among the low-level image features, the texture is robust and objective in CBIR. Various techniques for extracting texture features have been developed, which are generally classified as spatial and spectral 
methods. Spectral approaches of texture analysis for image retrieval are robust to noise. The spectral approaches include Fourier transform, multiresolution methods such as Gabor filters, and wavelet transform for texture representation [9]. The disadvantage of these spectral methods is that they do not effectively capture image edge information. This is the reason for finding better spectral approaches that can effectively capture an image's edge and orientation detail. The rest of the paper is organized as follows: Section II related work. Section III covers curvelet transform. Section IV, We explain the proposed methodology. Section V shows the results of performance evolution. Finally, section VI concludes this paper.

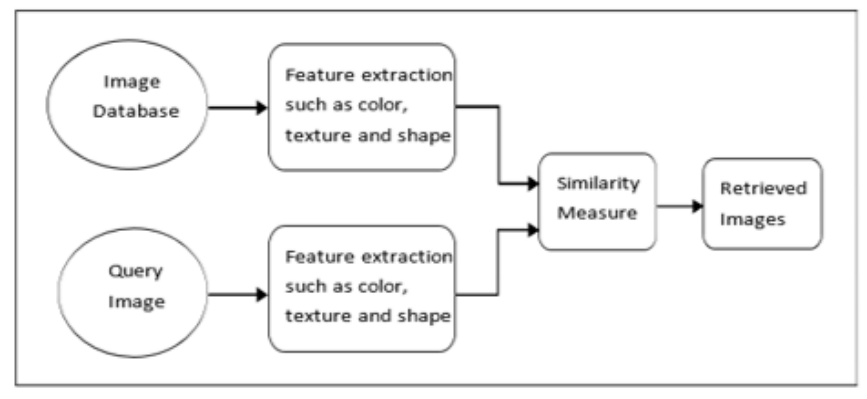

Figure 1: Content-based image retrieval (CBIR) system

\section{THE RELATED WORK}

This section presents some research on CBIR and curvelet transformation. These researchers have developed and improved the most significant research in this field: K. Jemseera and P Noufal in 2015 [10] proposed an enhanced satellite-based image fusion method based on the fast and discrete transformation of curvature (FDCT) through the wrapping. Satellite image fusion utilizes high-resolution panchromatic images and low-resolution multi-spectral images. The experimental results demonstrate that FDCT-based image fusion with an enhanced fusion rule produces a better spatial and spectral performance fused image than other fusion techniques. Amita et al. in 2017 [11] proposed a method for fast discrete curvelet transform-based anisotropic feature extraction in biomedical image indexing and retrieval. The curvelet transform is implemented in the image. The feature vector is determined using the directional energies of the curvelet coefficients, resulting in more directional information extracted at each scale. The efficacy of the suggested methodology is tested in three landmark biomedical databases. The results revealed a significant improvement in performance and computation time compared to other methods. In Baharudin et al. [12] have proposed a system using the query sub-blocks and database images, the adjacency matrix of a bipartite graph is formed. Extraction of statistical textural features from the quantized histograms in the discrete cosine transform employing only the DC coefficient and three AC coefficients for effective image retrieval. Guo et al. [13] suggested a novel approach to image indexing based on features derived from error diffusion block truncation coding (EDBTC). To evaluate the similarity between a query image and the image in the database, Bit Pattern Histogram Feature (BHF) and two features Color Histogram Feature (CHF) are introduced. The experimental results indicate that the proposed system outperforms previous BTC-based image indexing and other current image retrieval schemes. The EDBTC has well capable of image compression as well as indexing images for CBIR. Guo et al. [14] have developed a 
hybrid retrieval system that uses a Local binary pattern (LBP) as a texture extraction technique. Here, the LBP calculation is based on the different scales, which capture more prominent features of an image as compared to single-scale LBP. Lastly, the Gray level co-occurrence matrix (GLCM) has been used efficiently to compute feature vectors. Yogita D. Mistry in 2020 [15] proposes a CBIR approach that uses a mixture of various textural and color picture descriptors, such as Gabor wavelet texture features and a fuzzy histogram, to achieve the highest precision. The Laplacian score is used as a feature selection method to pick appropriate features while discarding redundant ones. For the final image retrieval application, distance metrics are used to calculate similarity.

\section{iII. Curvelet Transform}

A multiresolution transform is known as Curvelet Transform developed by Candes and Donoho, which shows an advancement to overcome the limitations of wavelet and Gabor filters [16]. Curvelet transform has been developed to achieve complete coverage of the spectral domain and to capture more orientation information, The first generation of curvelets was based on Ridgelets transform [17], but due to its slow speed and high amount of redundancy, it was discarded in the second generation of curvelets in which Candes et al. proposed two new forms of curvelet transform based on different Fourier sample processes [18] , namely, unequally-spaced fast Fourier transform (USFFT) and wrapping based fast curvelet transform. Wrapping-based curvelet transform is faster in computation time and more robust than ridgelet and USFFT based curvelet transform [19] . FDCT based on the wrapping of Fourier samples has less computational complexity as it uses fast Fourier transform instead of complex ridgelet transform [20]. The curvelet transform, which is based on the wrapping of Fourier sampling, takes a 2-D image in the form of a Cartesian array $f[m, n]$ such that $0<n \leq N-1$, $0 \leq m<M-1$ as an input and generates several curvelet coefficients. Discrete curvelet coefficients can be defined by [21] :

$$
C_{j, \Theta}(k, 1)=\sum_{n=0}^{N-1} \sum_{m=0}^{M-1} f(x, y) \Psi_{j, \Theta, k, 1}(m, n)
$$

where $\Psi_{j, \Theta, k, l}(m, n)$ is a discrete curvelet, $\mathrm{j}$ and $\Theta$ are the scale and orientation respectively, $\mathrm{k}$ and $\mathrm{l}$ are the spatial location parameters. A wedge is a curvelet's frequency response, and the curvelet tiling of the frequency plane that covers the entire image in the spectral domain is shown in Fig. 2.

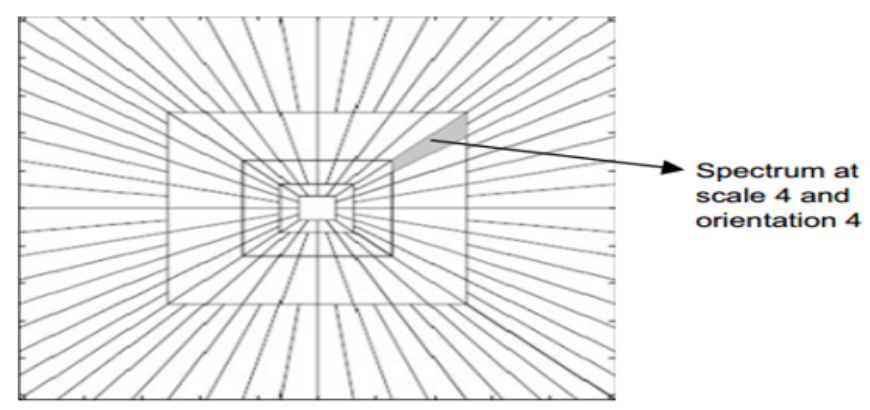

Figure 2: Curvelet tiling of frequency plane with 5 level curvelets 
Iraqi Journal of Information and Communications Technology(IJICT)

Conference Series: The $1^{\text {st }}$ Conference of Applied Researches

in Information Engineering(ARIE2021), 2021

ISSN:2222-758X

e-ISSN:2789-7362

Curvelet transform is applied in the frequency domain to achieve a higher level of efficiency and effectiveness. The image and the curvelet are both transformed and multiplied in the Fourier frequency domain. The result is then inverse Fourier transformed to obtain the curvelet coefficients. The process can be summarized as follows:

$$
\text { CurveletTransform }(\text { Image })=F F T-1(F F T(\text { Curvelet }) \times F F T(\text { Image }))
$$

However, the product from the multiplication is a wedge. The properties of the curvelet can be summarized as follows [22]:

1) The transform curvelet is based on a modern type of pyramid filter structure.

2) The frame elements of the curvelet include new scaling rules.

3) Curvelet provides an adequate representation of images with edges that curvelet can provide.

\section{METHODOLOGY}

This section explains the details of each stage of the proposed system. The proposed system consists of three stages: preprocessing to remove the noise from the image, feature extraction to extract the essential properties of an image using Curvelet Transform, and the similarity measurement to retrieve the similar image from the image dataset.

\section{A. Pre-Processing}

The initial stage in the proposed system is the preprocessing algorithm. Image quality measurement is an essential task for many applications of image processing. The quality is based on the differences or similarities between a distorted image and the original or unmodified image. Noise removal from the images is achieved using different filters such as Mean filter, Median filter, and Gaussian filter. MATLAB 2017b is designed to implement codes. Two noises have been considered in this work: salt \& pepper and AWGN. At the final step, the results of the mentioned filters were compared to get the suitable filter for these images. The results verified that the median filter is a suitable filter to remove the noise in these images. Fig. 3 shows the block diagram of the preprocessing stage.

\section{B. Curvelet Texture Features Extraction}

A features extraction method is based on Curvelet transform, which is proposed and presented. Discrete curvelet transform is implemented using the wrapping-based fast discrete curvelet transform. The image and the curvelet are both transformed into the Fourier domain at a given scale and orientation. The convolution of the curvelet with an image in the spatial domain then becomes their result in the Fourier domain. At the end of these computation steps, we obtain a set of curvelet coefficients by implementing inverse FFT to the spectral product. Fig. 4 depicts the entire feature extraction process using a single curvelet. The Curvelet decomposition generates several sub-bands images, then statistical features from this sub-band are calculated to generate a feature vector of the image. In this work, the image is decomposed into three, four, five, six, and seven scales. The number of the directional sub-band images varies from one scale to another. After the curvelet coefficients are generated and stored in each subband, the mean and standard deviation of the coefficients associated with each subband is computed. Generally, these mean and standard deviations are then used as the texture feature vector elements of the 
Iraqi Journal of Information and Communications Technology(IJICT)

image. Thus, we obtain two texture features for each curvelet. These feature descriptors are used to index images in the feature database, also known as the image 'indexing scheme'.

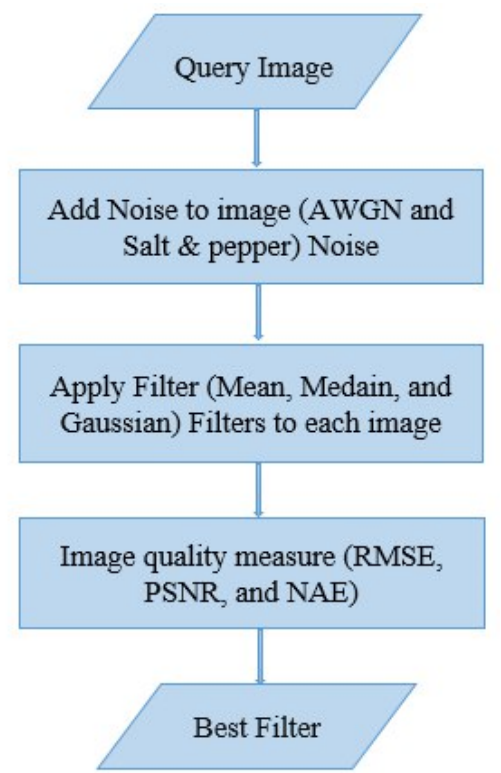

Figure 3: Block diagram of preprocessing stage

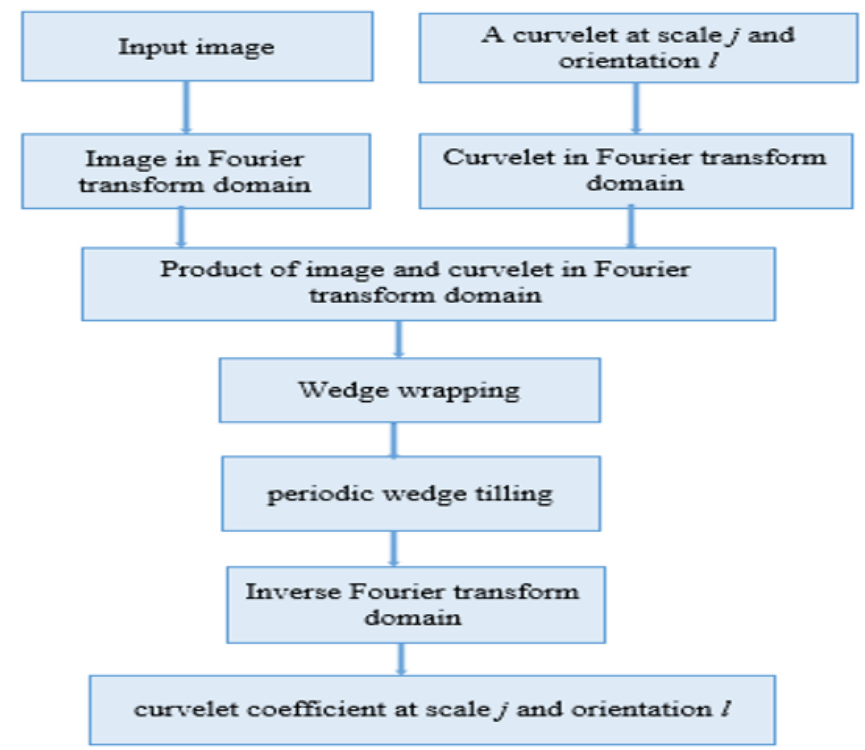

Figure 4: FDCT to generate curvelet coefficients 


\section{Similarity Measure}

The similarities between the query images and the characteristics of the images held in the database are measured by Euclidean distance. The Euclidean distance method is the distance between two points that can be measured and produced based on two vectors. Based on the two vectors, the distance between the two vectors can be determined using the following equation [23] :

$$
E D(Q, T)=\sum_{i=1}^{2 n}\left(Q_{i}-T_{i}\right)^{1 / 2}
$$

Here, $Q=\left\{Q_{1}, Q_{2}, \ldots, Q_{2 n}\right\}$ is the feature vector of the query image, and $T=\left\{T_{1}, T_{2}, \ldots, T_{2 n}\right\}$ is the feature vector of the target image in the database.

\section{Experiments}

The experiments were implemented on a database comprising 1000 diverse images, and each image in the dataset is $384 * 256$ or $256 * 384$ size-wise taken from the coral database. There are ten different categories, and each category contains one hundred images. This results in an extensive database of 1000 images and all the images in JPEG format. The Corel dataset is of the following semantic categories contain Mountains, Africa, Dinosaurs, Buildings, Buses, Food, Elephants, Horses, beaches, and Flowers. These semantic categories were used to evaluate retrieval performance because the majority of the noteworthy studies utilized these groups in their demonstration of the effectiveness of their CBIR methods. Some of the different images from 10 semantic sets of the Corel dataset are shown in Fig. 5.

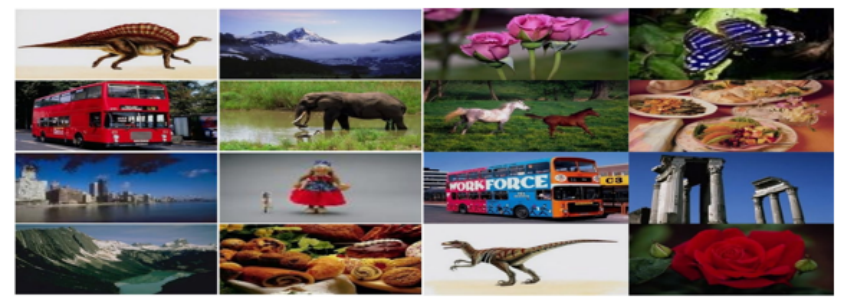

Figure 5: Simple image from the coral database

We applied the curvelet feature extraction process to each of the 1000 images in the database and index each of the images using the curvelet feature vector. For the discrete curvelet transform, three tests were experimented with. In the first test, images are decomposed using three levels of curvelet transform. Eighteen subbands of curvelet coefficients are computed. In the second test, images are decomposed using four levels of curvelet transforms, and 52 subbands of curvelet coefficients have been computed. In the third test, images are decomposed using five levels of curvelet transforms, and 82 subbands of curvelet coefficients have been computed, and so in for another level are shown in Table I. After that, we calculate the mean and standard deviation of the first half of the total subbands at each scale (excluding the subband at the coarsest and the finest level are calculated separately) because that the curvelet at angle $\theta$ produces the same coefficients as the curvelet at angle $\theta+\pi$. Therefore, half of the subbands are discarded due to this symmetry. As for the feature vector, all 
these elements are organized in it so that the standard deviations remain in the first half of the feature vector and the means are inserted into the second half of the feature vector. Where we obtain a total of $(1+8+1)=10$ subbands for each of the images in the database when using 3 levels of discrete curvelet decomposition. Hence the feature vector for each image becomes $10 \times 2=20$ dimensional. When using 4 levels of discrete curvelet decomposition, we get $(1+8+16+1)=26$ subbands for each image in the database. The feature vector for each image becomes $26 \times 2=52$ dimensional and so in for another level are shown in Table I. With these steps, a features vector is generated for each image in the database of each level decomposition. The extracted features were all assembled and merged within the created features repository. Following the extraction of features from database images, a comparison was made between the input query images and related images in the features repository using Euclidean distance. Precision and recall pair is used to evaluate retrieval performance. Precision $\mathrm{P}$ is defined as a ratio of the number of relevant images retrieved $\mathrm{r}$ to the total number of images retrieved n, i.e., $P=r / n$. Precision $\mathrm{P}$ measures the accuracy of the retrieval. Recall $\mathrm{R}$ is defined as a ratio of the number of retrieved relevant images $\mathrm{r}$ to the total number of relevant images $\mathrm{m}$ in the entire database, i.e., $R=r / m$. Recall $\mathrm{R}$ measures the robustness of the retrieval.

TABLE I

The Feature Vector of Curvelet Transform

\begin{tabular}{|c|c|c|}
\hline & Total no. sub bands & No. of the feature vector \\
\hline Level 3 & 18 & 20 \\
\hline Level 4 & 50 & 52 \\
\hline Level 5 & 82 & 84 \\
\hline Level 6 & 146 & 148 \\
\hline Level 7 & 210 & 212 \\
\hline
\end{tabular}

V. RESUlt

In this section, three types of filters have been used to remove noise and to enhance the quality of the input image. It was found that all filters could remove the noise and had successful results. To obtain the optimum filter on images, this depended on the image quality parameters of PSNR, RMSE and NAE. The median filter had a maximum value of PSNR, minimum values of NAE and RMSE. Therefore, the Median filter had more ability in removing noise. The results were listed in Tables II to IV. Each image in the database is used to serve as a query. For each query, the retrieval precision at each level of the recall is calculated. These precision values are then averaged to generate the average precision-recall curve for the database images. The curvelet transforms with seven levels decomposition have even better retrieval performance but takes more time. For this time evaluation, we have used the Matlab function called tic toc, which gives the total elapsed time to retrieve an image. Table $\mathrm{V}$ shows the elapsed time for the feature extraction and the average precision-recall. Table VI is evident that precision is improved using the curvelet transform method proposed in this article. The increase in the retrieved similar images will improve precision and recall rates. Result images displayed using GUI, which gives exact images or similar images that are shown in Fig. 6. Fig. 6 is a set of images retrieved as a result of applying a curved transformation with seven levels of decomposition to the query image. 
Iraqi Journal of Information and Communications Technology(IJICT)

TABLE II

The Result of Applying Three Filters to Images Corrupted with Gaussian Noise

\begin{tabular}{|c|c|c|c|c|c|c|}
\hline \multirow{2}{*}{$\begin{array}{c}\text { Image } \\
\text { Quality Measures }\end{array}$} & \multicolumn{2}{|c|}{ Average filter } & \multicolumn{2}{c|}{ Median filter } & \multicolumn{2}{c|}{ Gaussian filter } \\
\cline { 2 - 7 } & Image 1 & Image 2 & Image 1 & Image 2 & Image 1 & Image 2 \\
\hline RMSE & 15.522 & 14.086 & 13.849 & 12.261 & 14.816 & 13.899 \\
\hline PSNR & 44.657 & 42.563 & 44.862 & 43.941 & 44.453 & 43.144 \\
\hline NAE & 0.201 & 0.1 & 0.097 & 0.085 & 0.11 & 0.089 \\
\hline
\end{tabular}

TABLE III

The Results of Applying Three Filters to Images Corrupted with Salt and Pepper Noise

\begin{tabular}{|c|c|c|c|c|c|c|}
\hline \multirow{2}{*}{$\begin{array}{c}\text { Image } \\
\text { Quality Measures }\end{array}$} & \multicolumn{2}{|c|}{ Average filter } & \multicolumn{2}{c|}{ Median filter } & \multicolumn{2}{c|}{ Gaussian filter } \\
\cline { 2 - 7 } & Image 1 & Image 2 & Image 1 & Image 2 & Image 1 & Image 2 \\
\hline RMSE & 14.924 & 13.788 & 14.391 & 14.101 & 15.102 & 13.695 \\
\hline PSNR & 43.832 & 43.295 & 44.417 & 43.661 & 44.186 & 42.708 \\
\hline NAE & 0.102 & 0.074 & 0.079 & 0.063 & 0.084 & 0.182 \\
\hline
\end{tabular}

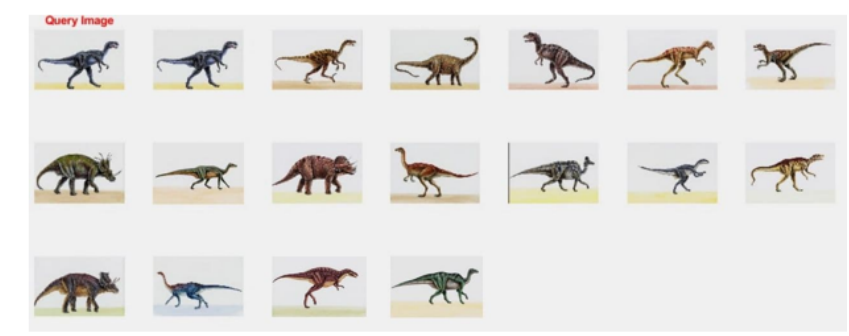

(a) Results of relevant images retrieved by inputting dinosaur image as a query image

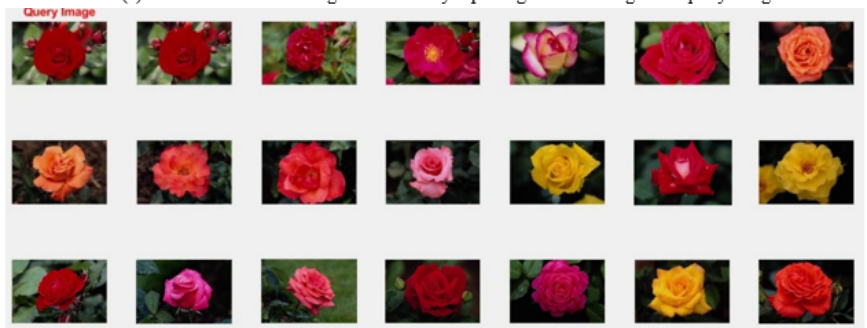

(b) Results of relevant images retrieved by inputting flower image as a query image

Figure 6: Some relevant images retrieved

TABLE IV

Result of Applying Three Filters to Real Images

\begin{tabular}{|c|c|c|c|c|c|c|}
\hline Image & \multicolumn{2}{|c|}{ Average filter } & \multicolumn{2}{c|}{ Median filter } & \multicolumn{2}{c|}{ Gaussian filter } \\
\cline { 2 - 7 } Quality Measures & Image 1 & Image 2 & Image 1 & Image 2 & Image 1 & Image 2 \\
\hline RMSE & 14.924 & 13.789 & 13.412 & 13.101 & 15.145 & 13.696 \\
\hline PSNR & 41.365 & 43.655 & 45.642 & 43.693 & 44.143 & 43.671 \\
\hline NAE & 0.012 & 0.015 & 0.009 & 0.01 & 0.002 & 0.04 \\
\hline
\end{tabular}

TABLE V

Retrieval Accuracy of Curvelet Feature

\begin{tabular}{|c|c|c|c|}
\hline & Total time for all images (seconds) & Recall (\%) & Precision (\%) \\
\hline Level 3 & 26.4111 & 36.41 & 79.64 \\
\hline Level 4 & 45.2135 & 39.70 & 85.23 \\
\hline Level 5 & 45.2372 & 58.29 & 89.75 \\
\hline Level 6 & 45.2835 & 62.02 & 92.69 \\
\hline Level 7 & 74.6012 & 89.26 & 99.21 \\
\hline
\end{tabular}


Iraqi Journal of Information and Communications Technology(IJICT)

Conference Series: The $1^{\text {st }}$ Conference of Applied Researches

TABLE VI

Average Precision Comparison of Various CBIR Methods Using Coral Dataset

\begin{tabular}{|c|c|c|c|c|c|}
\hline Method & Baharudin et al. [12] & Guo et al. [13] & Guo et al. [14] & Yogita D. Mistry [15] & Proposed Method \\
\hline Average Precision & 81 & 79.7 & 77.3 & 87.7 & 89.3 \\
\hline
\end{tabular}

\section{Conclusion}

This paper developed a mechanism for automatic image retrieval based on Curvelet Transform. We have been applied types of filters that significantly impact the images. However, the filter choice depends on several image quality measures such as RMSE, PSNR, and NAE. Results show the efficiency of the median filter in the reduction of the noise. We used more appropriate Texture features to form a feature vector. Then we compare our query image with all the database images using Euclidean distance. The proposed system has a significant level of efficiency for the retrieval of images by obtaining higher precision and recall values, and it seems that this system has better performance than other methods. This application may use in internet image searching, Bio-medical image retrieval in high semantic meaning, decision support systems, and accurate image retrieval from extensive image archives. 
Iraqi Journal of Information and Communications Technology(IJICT)

Conference Series: The $1^{\text {st }}$ Conference of Applied Researches

in Information Engineering(ARIE2021), 2021

ISSN:2222-758X

e-ISSN:2789-7362

\section{REFERENCES}

[1] Z. Li, X. Zhang, H. Muller, S. Zhang, "Large Scale Retrieval for Medical Image Analytics: A Comprehensive Review" , Med Image Anal, Vol. 43, pp. 66-84, 2018.

[2] W. Huang, Y. Gao, KL. Chan, "A Review of Region Based Image Retrieval" , Signal Process Syst. , Vol. 59, No. 2, pp.143-161, 2010.

[3] D. Kurchaniya and P. K. Johari, "Analysis of Different Similarity Measures in Image Retrieval Based on Texture and Shape" , International Research Journal of Engineering and Technology (IRJET), Vol. 4, No. 4, pp. 2137-2143, 2017.

[4] T. Vipin, "Content Based Image Retrieval: Ideas, Influences, and Current Trends" , pp. 3-4, P. 7, 2018.

[5] S. Bhattacharyya, H. Hrishikesh, S. De, G. Klepac,"Intelligent Analysis of Multimedia Information" , p. 145, 2016.

[6] G. Osvaldo, M. Beniamino, M. Sanjay, S. Elena, M. Carmel, A.C. Ana Maria, T. David, O. Bernady, T. Eufemia, R. Yeonseung, "Computational Science and Its Applications-ICCSA", 18th International Conference, Melbourne, VIC, Australia, July, Proceedings, Part IV, p. 512, pp. 2-5, 2018.

[7] R Ashraf, M Ahmed, S Jabbar, S Khalid, A Ahmad, S Din, G Jeon, "Content Based Image Retrieval by Using Color Descriptor and Discrete Wavelet Transform", J Med Syst. , Vol. 42, No. 3, pp. 1-44, 2018.

[8] R. Ashraf, K. Bashir, MT. Mahmood, A. Irtaza, "Content Based Image Retrieval Using Embedded Neural Networks with Bandletized Regions" , Entropy, Vol. 17, No. 6, pp. 3552-3580, 2015.

[9] G. Osvaldo, M. Beniamino, M. Sanjay, S. Elena, M. Carmel, A.C. Ana Maria, T. David, O. Bernady, T. Eufemia, R. Yeonseung, "Computational Science and Its Applications-ICCSA", 18th International Conference, Melbourne, VIC, Australia, Proceedings, Part IV, pp. 2-5, July, 2018.

[10] K. Jemseera and P Noufal, "Satellite Image Fusion Based on Improved Fast Discrete Curvelet Transforms", Fifth International Conference on Advances in Computing and Communications, 2015.

[11] A. Amita, D. Amol, Y. Chetankumar, "Fast Discrete Curvelet Transform Based Anisotropic Feature Extraction for Biomedical Image Indexing and Retrieval" , 2017.

[12] G. Papakostas, D. Koulouriotis, and V. Tourassis, "Feature Extraction Based on Wavelet Moments and Moment Invariants in Machine Vision Systems" , in Human-Centric Machine Vision, InTech, London, UK, 2012.

[13] J. M. Guo, H. Prasetyo, and H. Su, "Image Indexing Using The Color and Bit Pattern Feature Fusion" , J. Vis. Commun. Image Represent, Vol. 24, No. 8, pp. 1360-1379, 2013.

[14] J. M. Guo, H. Prasetyo, and J. H. Chen, "Content Based Image Retrieval Using Error Diffusion Block Truncation Coding Features" , IEEE Transactions on Circuits and Systems for Video Technology, Vol. 25, No. 3, pp. 466-481, 2015.

[15] D. Yogita Mistry, "Textural and Color Descriptor Fusion for Efficient Content Based Image Retrieval Algorithm" , 2020, https://doi.org/10.1007/s42044-020-00056-0.

[16] E. Candes, L. Demanet, D. Donoho, and L. Ying, "Fast Discrete Curvelet Transforms" , Multiscale Modelling and Simulation, Vol. 5, No. 3 pp. 861-899, 2006.

[17] K. M. Jyotsna, B. Kallol, I. Majumdar, M. Surajit, "Information, Photonics, and Communication: Proceedings of Second National Conference" , IPC, p. 186, 2019.

[18] E. J. Candes, L. Demanet, L. Ying, and D. L. Donoho, "Fast Discrete Curvelet Transforms" , Multiscale Modeling and Simulation, Vol. 5, pp. 861-899, 2005.

[19] M. J. Fadili and J. Starck, "Curvelets and Ridgelets" , Encyclopedia of Complexity and System Science, in press. , 2007.

[20] H. S. Saini, S. Rishi, A. Govardhan, B. Rajkumar," Innovations in Computer Science and Engineering" , Proceedings of the Fifth ICICSE 2017, pp. 16-31, 2018.

[21] B. Pongsarun, M. Phayung, S. Sunantha, U. Herwig, "Analyze Facial Expression Recognition Based on Curvelet Transform via Extreme Learning Machine" , Recent Advances in Information and Communication Technology, Proceedings of the 15th International Conference on Computing and Information Technology (IC2IT 2019), p. 150, 2019.

[22] B. Asim, H. Cresswell, "Curvelet Transform to Study Scale Dependent Anisotropic Soil Spatial Variation" , Elsevier Geoderma 213, PP. 589-599, 2014.

[23] D. Kurchaniya and P. K. Johari, "Analysis of Different Similarity Measures in Image Retrieval Based on Texture and Shape" , International Research Journal of Engineering and Technology (IRJET), Vol. 4, No. 4, pp. 2137-2143, 2017. 